M. Nakai

Nagoya Math. J.

Vol. 56 (1974), 105-119

\title{
A TEST FOR PICARD PRINCIPLE
}

\author{
MITSURU NAKAI
}

A nonnegative locally Hölder continuous function $P(z)$ on $0<|z| \leq 1$ will be referred to as a density on $0<|z| \leq 1$. The elliptic dimension of a density $P(z)$ at $z=0, \operatorname{dim} P$ in notation, is defined to be the dimension of the half module of nonnegative solutions of the equation $\Delta u(z)$ $=P(z) u(z)$ on the punctured unit disk $\Omega: 0<|z|<1$ with boundary values zero on $|z|=1$. After Bouligand we say that the Picard principle is valid for a density $P$ at $z=0$ if $\operatorname{dim} P=1$. The purpose of this paper is to establish the following practical test:

THEOREM. The Picard principle is valid for a density $P(z)$ on $0<|z| \leq 1$ at $z=0$ if there exists a closed subset $E$ of $\Omega$ such that $\Omega-E$ is connected and $z=0$ is an irregular boundary point of the region $\Omega-E$ for the harmonic Dirichlet problem and

$$
\int_{\Omega-E} P(z) \log \frac{1}{|z|} d x d y<\infty .
$$

As a direct consequence of the theorem we see that if $P \in L^{p}(\Omega-E)$ $(1<p \leq \infty)$ for an admissible exceptional set $E$ as stated in the theorem, then the Picard principle is valid for $P$. Needless to say, here and also in the theorem the exceptional set $E$ may be empty. We must also remark that (1) is not necessary for the validity of Picard principle as is seen by a simple example $P(z)=|z|^{-2}$ (cf. no. 12). The proof of the theorem will be given in nos. 9-8. In the last no. 12 we state four unsettled important problems related to elliptic dimensions.

1. Let $P(z)$ be a density on $0<|z| \leq 1$, i.e. $P(z) \geq 0$ and $P(z)$ is locally Hölder continuous: $\left|P\left(z_{1}\right)-P\left(z_{2}\right)\right| \leq A_{r}\left|z_{1}-z_{2}\right|^{\alpha_{r}}$ for every $z_{1}$ and $z_{2}$ in $0<r \leq|z| \leq 1$ where $A_{r} \in(0, \infty)$ and $\lambda_{r} \in(0,1]$ are constants which may depend on $r \in(0,1)$. Such a density $P$ can be considered to be a density on $\Omega: 0<|z|<1$ which is the restriction to $\Omega$ of a density on

Received March 22, 1974. 
$\hat{\Omega}: 0<|z|<\infty$. Let $\hat{P}(z)$ be the symmetric extension of a density $P(z)$ on $0<|z| \leq 1$ to $\hat{\Omega}: \hat{P}(z)=P(z)$ on $\Omega$ and $\hat{P}(z)=P(1 / \bar{z})$ for $1 \leq|z|<\infty$. Then $\hat{P}(z)$ is a density on $\hat{\Omega}$ and $\hat{P} \circ \tau=\hat{P}$, where $\tau$ is the involution of $\hat{\Omega}$ about $|z|=1$, i.e. $\tau(z)=1 / \bar{z}$.

The basic tool of our proof is the unique solvability of the Dirichlet problem. Let $R$ be a region in $\hat{\Omega}$ bounded by a finite number of disjoint analytic Jordan curves and $Q$ be a density on $\hat{\Omega}$. For any $\varphi \in C(\partial R)$ there exists a unique function $Q_{\varphi}^{R} \in C(\bar{R})$ such that $Q_{\varphi}^{R}=\varphi$ on $\partial R$ and $Q_{\varphi}^{R}$ is a solution of $\Delta u=Q u$ on $R$. If $Q \equiv 0$, then we use the standard notation $H_{\varphi}^{R}$ instead of $Q_{\varphi}^{R}$. The unique existence of $H_{\varphi}^{R}$ can be seen e.g. by the Perron-Brelot method as can be found in any text book (cf. e.g. Tsuji [18]). By the same method we can see the unique existence of $Q_{\phi}^{R}$ but the following integral equation method is preferable for our purposes in the sense that it clarifies the relation between $Q_{\varphi}^{R}$ and $H_{\varphi}^{R}$. Let $\mathrm{H}_{R}(z, \zeta)$ be the harmonic Green's function on $R$ (cf. e.g. [18]) and consider the integral operator

$$
(T f)(z)=-\frac{1}{2 \pi} \int_{R} H_{R}(z, \zeta) Q(\zeta) f(\zeta) d \xi d \eta \quad(\zeta=\xi+i \eta) .
$$

It is elementary to check that $f \in C^{\alpha}(D)$ implies $T f \in C^{\alpha+1}(D) \quad(\alpha=0,1)$ and $\Delta T f=Q \cdot f$ on $D(\alpha=1)$ for an open set $D$ in $R$ and for an $f$ on $R$ for which $T f$ can be defined (cf. e. g. Miranda [9]). It is also easy to see that $f \in C(\bar{R})$ implies $T f \in C(\bar{R})$ with $T f=0$ on $\partial R$, and that $T$ is a compact operator from $C(\bar{R})$ into itself. By the maximum principle for subharmonic functions we see that 1 is not the proper value of $T$ and therefore by the Riesz-Schauder theory, $I+T: C(\bar{R}) \rightarrow C(\bar{R})$ is bijective (cf. e.g. Yosida [19]). Hence $Q_{\varphi}^{R}$ is obtained as $(I+T)^{-1} H_{\varphi}^{R}$ :

$$
Q_{\varphi}^{R}(z)=H_{\varphi}^{R}(z)-\frac{1}{2 \pi} \int_{R} H_{R}(z, \zeta) Q(\zeta) Q_{\varphi}^{R}(\zeta) d \xi d \eta .
$$

By the fact that $\varphi \rightarrow H_{\varphi}^{R}$ is a positive linear operator from $C(\partial R)$ into $C(\bar{R})$ with norm 1 and by the maximum principle of subharmonic functions, we see that $\varphi \rightarrow Q_{\varphi}^{R}$ is a positive linear operator from $C(\partial R)$ into $C(\bar{R})$ with norm 1.

Fix a $\zeta_{0} \in R$ and let $R_{n}$ be the region obtained from $R$ by deleting the closed disk about $\zeta_{0}$ with radius $1 / n$ for large integer $n$ and $T_{n}$ be the corresponding integral operator: $C\left(\bar{R}_{n}\right) \rightarrow C\left(\bar{R}_{n}\right)$. Then $u_{n}=\left(I_{n}\right.$ 
$\left.+T_{n}\right)^{-1} H_{R}\left(\cdot, \zeta_{0}\right)$ forms a decreasing sequence dominated by $H_{R}\left(\cdot, \zeta_{0}\right)$, and if we denote by $G_{R}\left(\cdot, \zeta_{0}\right)$ the limit function, then

$$
G_{R}\left(z, \zeta_{0}\right)=H_{R}\left(z, \zeta_{0}\right)-\frac{1}{2 \pi} \int_{R} H_{R}(z, \zeta) Q(\zeta) G_{R}\left(\zeta, \zeta_{0}\right) d \xi d \eta
$$

The function $G_{R}(z, \zeta)$ is referred to as the Green's function of $\Delta u=Q u$ on $R$. By (3) we see that $G_{R}(\cdot, \zeta) \in C^{1}(\bar{R}-\{\zeta\})$ and $\partial G_{R}(z, \zeta) / \partial t$ $=\partial H_{R}(z, \zeta) / \partial t+O(1)$ as $z \rightarrow \zeta$ where $t=x$ and $y$. By this and by the Green formula we have the symmetry $G_{R}(z, \zeta)=G_{R}(\zeta, z)$. We denote by $C^{\omega}(\partial R)$ the class of real analytic functions on $\partial R$. If $\varphi \in C^{\omega}(\partial R)$, then $H_{\varphi}^{R}$ is easily seen to belong to $C^{1}(\bar{R})$, and by (2) we see that $Q_{\varphi}^{R} \in C^{1}(\bar{R})$. By the Green formula

$$
Q_{\varphi}^{R}(z)=\frac{1}{2 \pi} \int_{\partial R} \varphi(\zeta) \frac{\partial}{\partial \nu_{\zeta}} G_{R}(z, \zeta) d s_{\zeta}
$$

where $\partial / \partial \nu$ denotes the inner normal derivative and $d s$ is the line element. This is primarily derived for $\varphi \in C^{\omega}(\partial R)$ but the denseness of $C^{\omega}(\partial R)$ in $C(\partial R)$ assures the validity of (4) for every $\varphi \in C(\partial R)$. As a consequence of (4) we have the Harnack inequality and the Harnack principle for nonnegative solutions of $\Delta u=Q u$.

2. For a density $P(z)$ on $0<|z| \leq 1$ we shall study the half module $\mathscr{P}$ of nonnegative solutions $u$ of the equation $\Delta u(z)=P(z) u(z)$ on the punctured disk $\Omega: 0<|z|<1$ with boundary values zero on $\beta:|z|=1$. For the study of $\mathscr{P}$ we need to consider the half module $\mathscr{B}$ of nonnegative bounded solutions of $\Delta u=P u$ on $\Omega$ with continuous boundary values on $\beta$. Let $\Omega_{t}$ be $0<|z|<t$ and $\beta_{t}$ be $|z|=t$ for $t \in(0,1]$. Thus $\Omega_{1}=\Omega$ and $\beta_{1}=\beta$. We also consider auxiliary classes $\mathscr{P}_{t}$ and $\mathscr{B}_{t}$ of nonnegative and nonnegative bounded solutions of $\Delta u=P u$ on $\Omega_{t}$ with boundary values zero and continuous boundary values on $\beta$, respectively. In particular $\mathscr{P}_{1}=\mathscr{P}$ and $\mathscr{B}_{1}=\mathscr{B}$. The boundary point $z=0$ is of parabolic character (cf. Brelot [1], Ozawa [14], Royden [17]) in the following sense:

$$
\mathscr{P}_{t} \cap \mathscr{B}_{t}=\{0\} .
$$

Let $u \in \mathscr{P}_{t} \cap \mathscr{B}_{t}$. Since $\Delta u=P u \geq 0, u$ is subharmonic on $\Omega_{t}$. For any $\varepsilon>0 s_{\varepsilon}(z)=-\varepsilon \log |z|-u(z)$ is superharmonic on $\Omega_{t}$ with $\lim \inf _{z \rightarrow \partial \Omega_{t}} s_{\varepsilon}(z)$ $\geq 0$. The minimum principle for superharmonic functions yields $s_{s}(z)$ $\geq 0$ for every $\varepsilon>0$ and therefore $u=0$ on $\Omega_{t}$. For any $u \in \mathscr{B}_{t}$ let $u_{t, s}$ 
be the solution of $\Delta u=P u$ on $\Omega_{t}-\bar{\Omega}_{s}(0<s<t \leq 1)$ with boundary values $u$ on $\beta_{t}$ and zero on $\beta_{s}$. As a consequence of (5) we have

$$
u(z)=\lim _{s \rightarrow 0} u_{t, s}(z)
$$

on $\Omega_{t}$. In fact, let $v=\lim _{s \rightarrow 0} u_{t, s} \in \mathscr{B}_{t}$. Then $0 \leq v \leq u$ on $\Omega_{t}$ with $v=u$ on $\beta_{t}$. Thus $u-v \in \mathscr{P}_{t} \cap \mathscr{B}_{t}$, i.e. (6) is valid. Therefore $u \in \mathscr{B}_{t}$ is determined uniquely by its boundary values $\varphi$ on $\beta_{t}$. We shall denote this $u$ by $P_{\varphi}^{\Omega_{t}}$. Then $\varphi \rightarrow P_{\varphi}^{\Omega_{t}}$ is a positive linear operator from $C\left(\beta_{t}\right)$ into $\mathscr{B}_{t} \ominus \mathscr{B}_{t}$ with norm 1 .

Fix a $t$ and an $s$ with $0<s<t \leq 1$. Consider the operator $S$ $=S_{s, t}$ from $\mathscr{P}_{t}$ into $\mathscr{P}_{s}$ given by $S u=u-P_{u}^{o_{t}}$. Then $S$ is a bijective half linear operator between $\mathscr{P}_{t}$ and $\mathscr{P}_{s}$ (Heins [4], Ozawa $\left.(15,16]\right)$. If $S u=0$, then $u=P_{u}^{\Omega_{s}}$ and $u$ is bounded, i.e. $u \in \mathscr{P}_{t} \cap \mathscr{B}_{t}$ and $u=0$. Thus $S$ is injective. Let $v \in \mathscr{P}_{s}$ and $u_{r}=P_{v}^{g_{t}-\Omega_{r}}$ where $\bar{v}=v$ on $\beta_{r}$ and $\bar{v}=0$ on $\beta_{t}(0<r<s)$ and $w_{r}=u_{r}-v \geq 0$. Since $\left\{u_{r}\right\}$ is increasing as $r \rightarrow 0$, if $u=\lim _{r \rightarrow 0} u_{r}$ is convergent, then $u \in \mathscr{P}_{t}$ and $\lim _{r \rightarrow 0} w_{r}=P_{u}^{\Omega_{s}}$ and $S u$ $=v$, i.e. $S$ is surjective. Let $f_{t} u$ be the flux $\int_{0}^{2 \pi}\left[\partial u\left(r e^{i \theta}\right) / \partial r\right]_{r=t} t d \theta$ and $D_{A}(\varphi)=\int_{A}\left(|\nabla \varphi(z)|^{2}+P(z) \varphi(z)^{2}\right) d x d y$ where $\nabla \varphi=\left(\varphi_{x}, \varphi_{y}\right)$. Then by the Green formula

$$
f_{r} u_{r}-f_{t} u_{r}=D_{a_{t}-\bar{\Omega}_{r}}\left(u_{r}\right), \quad f_{r} v-f_{s} v=D_{\Omega_{s}-\bar{g}_{r}}(v)=D_{\Omega_{t}-\bar{\Omega}_{r}}(v)
$$

where we set $v=0$ on $\Omega_{t}-\Omega_{s}$. Again by the Green formula we see the Dirichlet principle: $D_{\Omega_{t}-\bar{\Omega}_{r}}\left(u_{r}\right) \leq D_{\Omega_{t}-\bar{\Omega}_{r}}(v)$. Hence

$$
0 \leq-f_{t} u_{r} \leq-f_{s} v-f_{r}\left(u_{r}-v\right)
$$

Since $u_{r}-v \geq 0$ and $u_{r}-v=0$ on $\beta_{r}$, we have $f_{r}\left(u_{r}-v\right) \geq 0$. Therefore

$$
0 \leq \lim _{r \rightarrow 0} \sup \left(-f_{t} u_{r}\right) \leq-f_{s} v<\infty
$$

and $\lim _{r \rightarrow 0} u_{r}=\infty$ does not hold and thus $u=\lim _{r \rightarrow 0} u_{r}$ is convergent. This means that $\operatorname{dim} P$ depends only on the behavior of $P$ at $z=0$.

Another consequence of (5) and actually of (6) is

$$
\int_{\Omega_{s}}|\nabla u(z)|^{2} d x d y<\infty
$$

for every $u \in \mathscr{B}_{t}$ and $s \in(0, t)$. This will play the essential role in the 
next no. 3. For $0<r^{\prime}<r<s$, the Dirichlet principle which is a simple consequence of the Green formula yields $D_{\Omega_{s}}\left(u_{s, r}\right)=D_{\Omega_{s}-\bar{\Omega}_{r^{\prime}}}\left(u_{s, r^{\prime}}\right) \leq D_{\Omega_{s}-\bar{g}_{r}}\left(u_{s, r}\right)$ $=D_{s_{s}}\left(u_{s, r}\right)$ where we have set $u_{s, r}=0$ on $\bar{\Omega}_{r}$ and $u_{s, r^{\prime}}=0$ on $\bar{\Omega}_{r^{\prime}}$. By (2) and (6) we have

$$
\lim _{r^{\prime} \rightarrow 0} \frac{\partial}{\partial a} u_{s, r^{\prime}}(z)=\frac{\partial}{\partial a} u(z)
$$

where $a=x$ and $y$, and by the Fatou lemma

$$
D_{\Omega_{s}}(u) \leq \lim _{r^{\prime} \rightarrow 0} \inf D_{\Omega_{s}}\left(u_{s, r^{\prime}}\right) \leq D_{\Omega_{s}}\left(u_{s, r}\right)<\infty
$$

for any fixed $r \in(0, s)$ and in particular we have (7).

3. The mean operation $u \rightarrow u^{*}$ is useful for the study of subharmonic functions. Let $u$ be defined on $\Omega_{t}$ such that

$$
u^{*}(r)=\frac{1}{2 \pi} \int_{0}^{2 \pi} u\left(r e^{i \theta}\right) d \theta
$$

can be defined for $r \in(0, t)$. This is the case e.g. when $u$ is subharmonic on $\Omega_{t}$. If $u$ is bounded subharmonic on $\Omega_{t}$, then $u$ can be extended to $|z|<t$ so as to be subharmonic by giving the value $\lim \sup _{z \rightarrow 0} u(z)$ at $z=0$, and hence we have (cf. e.g. Tsuji [18])

$$
\ell(u) \equiv \lim _{r \rightarrow 0} \frac{1}{2 \pi} \int_{0}^{2 \pi} u\left(r e^{i \theta}\right) d \theta=\lim _{z \rightarrow 0} \sup u(z)
$$

If $u \in \mathscr{B}_{t}$, then $u$ is bounded subharmonic on $\Omega_{t}$ and therefore the above relation (8) is applicable to every $u \in \mathscr{B}_{t}$.

We now maintain that for any $u \in \mathscr{B}_{t}$ there exists an exceptional closed subset $E=E_{u}$ of $(0, t)$ with finite logarithmic measure

$$
\int_{E} d \log r<\infty
$$

such that

$$
\lim _{|z| \notin E, z \rightarrow 0} u(z)=\ell(u) .
$$

For the proof consider Fourier coefficients $c_{n}(r)$ and $s_{n}(r)(n=1,2, \cdots)$ of $u\left(r e^{i \theta}\right)$ for any $r \in(0, t)$ as a function of $\theta$ : 


$$
\left\{\begin{array}{l}
c_{n}(r)=\frac{1}{\pi} \int_{0}^{2 \pi} u\left(r e^{i \theta}\right) \cos n \theta d \theta \\
s_{n}(r)=\frac{1}{\pi} \int_{0}^{2 \pi} u\left(r e^{i \theta}\right) \sin n \theta d \theta
\end{array}\right.
$$

for $n=1,2, \cdots$. On setting

$$
\varphi(r)=\left(\sum_{n=1}^{\infty} n^{2}\left(c_{n}(r)^{2}+s_{n}(r)^{2}\right)\right)^{1 / 2}
$$

we assert that

$$
\int_{0}^{1} \varphi(r) d \log r \leq \frac{1}{\pi} \int_{\Omega_{t}}|\nabla u(z)|^{2} d x d y<\infty .
$$

Observe that

$$
u\left(r e^{i \theta}\right)=u^{*}(r)+\sum_{n=1}^{\infty}\left(c_{n}(r) \cos n \theta+s_{n}(r) \sin n \theta\right)
$$

for $(r, \theta) \in(0, t) \times T$ with $T=(-\infty, \infty) / \bmod 2 \pi$ and thus

$$
u_{\theta}\left(r e^{i \theta}\right)=\sum_{n=1}^{\infty}\left(-n c_{n}(r) \sin n \theta+n s_{n}(r) \cos n \theta\right) .
$$

Therefore, in view of $\left|\nabla u\left(r e^{i \theta}\right)\right|^{2}=u_{r}\left(r e^{i \theta}\right)^{2}+r^{-2} u_{\theta}\left(r e^{i \theta}\right)^{2}$, we have

$$
r^{-2} \varphi(r)^{2}=\frac{1}{\pi} \int_{0}^{2 \pi} r^{-2} u_{\theta}\left(r e^{i \theta}\right)^{2} d \theta \leq \frac{1}{\pi} \int_{0}^{2 \pi}\left|\nabla u\left(r e^{i \theta}\right)\right|^{2} d \theta
$$

A fortiori

$$
\int_{0}^{t} \varphi(r)^{2} d \log r \leq \frac{1}{\pi} \int_{0}^{2 \pi} \int_{0}^{t}\left|\nabla u\left(r e^{i \theta}\right)\right|^{2} r d r d \theta,
$$

i.e. (11) is valid. Next set

$$
a_{n}=\int_{t /(n+2)}^{t /(n+1)} \varphi(r)^{2} d \log r
$$

for $n=1,2, \cdots$. By (11) we have $\sum_{n=1}^{\infty} a_{n}<\infty$. We can find a decreasing sequence $\left\{\varepsilon_{n}\right\}$ converging to zero such that $\sum_{n=1}^{\infty} \varepsilon_{n}^{-2} a_{n}<\infty$. Let

$$
E_{n}=\left\{r \in[t /(n+2), t /(n+1)] ; \varphi(r) \geq \varepsilon_{n}\right\}
$$

and

$$
E=E_{u}=\left(\bigcup_{n=1}^{\infty} E_{n}\right) \cap(0, t)
$$


which is a closed subset of $(0, t)$. Observe that

$$
\int_{E} d \log r=\sum_{n=1}^{\infty} \varepsilon_{n}^{-2} \int_{E_{n}} \varepsilon_{n}^{2} d \log r \leq \sum_{n=1}^{\infty} \varepsilon_{n}^{-2} \int_{E_{n}} \varphi(r)^{2} d \log r=\sum_{n=1}^{\infty} \varepsilon_{n}^{-2} a_{n},
$$

i.e. we have (9). By the Schwarz inequality

$$
\begin{aligned}
\left(u\left(r e^{i \theta}\right)-u^{*}(r)\right)^{2} & =\left(\sum_{n=1}^{\infty}\left(n c_{n}(r) \frac{\cos n \theta}{n}+n s_{n}(r) \frac{\sin n \theta}{n}\right)^{2}\right. \\
& \leq\left(\sum_{n=1}^{\infty} n^{-2}\right) \cdot\left(\sum_{n=1}^{\infty} n^{2}\left(c_{n}(r)^{2}+s_{n}(r)^{2}\right)\right) .
\end{aligned}
$$

Therefore we conclude that $\left|u\left(r e^{i \theta}\right)-u^{*}(r)\right| \leq 6^{-1 / 2} \pi \varphi(r)$. For an arbitrary $\varepsilon>0$ there exists by (8) an $r_{1} \in(0, t)$ such that $\left|u^{*}(r)-\ell(u)\right|<\varepsilon / 2$ for every $r \in\left(0, r_{1}\right)$. Let $n$ be such that $6^{-1 / 2} \pi \varepsilon_{n}<\varepsilon / 2$ and $\operatorname{set} r_{0}=\min \left(r_{1}, t /(n+1)\right)$. Then

$$
\left|u\left(r e^{i \theta}\right)-\ell(u)\right| \leq 6^{-1 / 2} \pi \varphi(r)+\left|u^{*}(r)-\ell(u)\right|<\varepsilon
$$

for every $r \in\left(0, r_{0}\right)-E$, i.e. we have (10).

4. The $P$-unit $e_{t}$ on $\Omega_{t}$ is the function in $\mathscr{B}_{t}$ with $e_{t} \mid \beta_{t}=1$. Using the $P$-unit $e=e_{1}$ on $\Omega=\Omega_{1}$ consider the equation

$$
\Delta v(z)+2 \nabla \log e(z) \cdot \nabla v(z)=0
$$

on $\Omega$. Let $v$ be a bounded nonnegative solution of (12) on $\Omega_{s}$. Then the following maximum-minimum principle is valid for $t \in(0, s)$ :

$$
\sup _{z \in \Omega_{t}} v(z)=\max _{z \in \beta_{t}} v(z), \quad \inf _{z \in \Omega_{t}} v(z)=\min _{z \in \beta_{t}} v(z) .
$$

By an easy computation one sees that $e v \in \mathscr{B}_{s}$. Let $c=\max _{\beta_{t}} v$ and $c^{\prime}=\min _{\beta_{t}} v$. Then since $e v, c e, c^{\prime} e \in \mathscr{B}_{t}$ and $c^{\prime} e \leq e v \leq c e$ on $\beta_{t}$, we see, by (6) or by the remark after (6) in no. 2, that $c^{\prime} e \leq e v \leq c e$ on $\Omega_{t}$ and thus $c^{\prime} \leq v \leq c$ on $\Omega_{t}$, i.e. (13) is valid.

5. Using results in nos. 3 and 4 we now maintain that under the assumption

$$
\ell(e)>0
$$

the following limit

$$
\lim _{z \rightarrow 0} u(z) / e(z)=\ell(u) / \ell(e)
$$

exists for every $u \in \mathscr{B}$. 
For the proof, let $E_{u}$ and $E_{e}$ be exceptional sets in no. 3 for $u$ and $e$ on $\Omega$, respectively. Then $E=E_{u} \cup E_{e}$ is also a closed subset of $(0,1)$ and (9) implies that

$$
\int_{E} d \log r<\infty .
$$

From this it follows that there exists a strictly decreasing sequence $\left\{r_{n}\right\}$ coverging to zero in $(0,1)-E$. Let $\varepsilon$ be an arbitrary number in $(0, \ell(e))$. By (10) there exists an $N$ such that

$$
|u(z)-\ell(u)|<\varepsilon, \quad|e(z)-\ell(e)|<\varepsilon
$$

for every $z \in \beta_{r_{n}}$ with $n>N$. Let $u_{e}=u / e$. Then again by an easy computation $u_{e}$ is a solution of (12). Since $u \leq c e$ on $\beta$ with $c=\max _{\beta} u$, (6) or the remark after (6) in no. 2 implies that $u_{e} \leq c$ on $\Omega$, i.e. $u_{e}$ is bounded on $\Omega$. Thus the maximum-minimum principle in no. 4 is applicable to $u_{e}$. Since

$$
\frac{\ell(u)-\varepsilon}{\ell(e)+\varepsilon} \leq u_{e}(z) \leq \frac{\ell(u)+\varepsilon}{\ell(e)-\varepsilon}
$$

on $\beta_{r_{n}}$, we have the same inequality on $\Omega_{r_{n}}$. Therefore

$$
\frac{\ell(u)-\varepsilon}{\ell(e)+\varepsilon} \leq \lim _{z \rightarrow 0} \inf \frac{u(z)}{\ell(z)} \leq \lim _{z \rightarrow 0} \sup \frac{u(z)}{\ell(z)} \leq \frac{\ell(u)+\varepsilon}{\ell(e)-\varepsilon}
$$

is valid for every $\varepsilon \in(0, \ell(e))$ and (15) follows.

6. Let $u$ be a continuous function on $\Omega \cup \beta$ such that $u$ is a solution of $\Delta u=P u$ on $\Omega$. Then the condition

$$
u\left(e^{i \theta}\right)=\left[\frac{\partial}{\partial r} u\left(r e^{i \theta}\right)\right]_{r=1}=0
$$

for every $\theta \in T=(-\infty, \infty) / \bmod 2 \pi$ implies that $u \equiv 0$ on $\Omega$.

Let $\hat{P}$ be the symmetric extension to $\hat{\Omega}$ of $P$ and fix a $t \in(0,1)$. Let $R$ be the annulus $t<|z|<1 / t$. Consider the solution $u_{1}$ of $\Delta u=\hat{P} u$ on $R$ with boundary values $u(z)$ on $|z|=t$ and $-u(1 / \bar{z})$ on $|z|=1 / t$. By the symmetry of $\hat{P}$ about $|z|=1, u_{1}(\tau(z))$ is also a solution of $\Delta u=P u$ where $\tau(z)=1 / \bar{z}$. Since $u_{1}(\tau(z))+u_{1}(z)=-u(1 /(1 / \bar{z}))+u(z)=0$ on $|z|=t$ and similarly on $|z|=1 / t$, we have $u_{1}(z)+u_{1}(\tau(z))=0$ on $R$ and in particular $u_{1}=0$ on $|z|=1$. Thus $u_{1}(z)=u(z)$ on $t \leq|z| \leq 1$. This means that $u$ has a $C^{2}$-extension to an open set containing $\Omega \cup \beta$. In 
particular $f(r)=r^{-2} u_{\theta \theta}\left(r e^{i \theta}\right)$ is continuous on $[t, 1]$ for any fixed $\theta \in T$ and the same is true of $g(r)=P\left(r e^{i \theta}\right)$. Consider the Cauchy problem for the linear ordinary equation

$$
\varphi^{\prime \prime}(r)+r^{-1} \varphi^{\prime}(r)+g(r) \varphi(r)=f(r)
$$

whose coefficients are continuous on $[t, 1]$ with the initial condition

$$
\varphi(1)=\varphi^{\prime}(1)=0
$$

on $[t, 1]$. Then $u\left(r e^{i \theta}\right)$, as a function of $r$, is a solution of this problem besides the trivial solution $\varphi(r) \equiv 0$. By the uniqueness of the solution of the Cauchy problem we have $u\left(r e^{i \theta}\right) \equiv 0$ on $[t, 1]$ for any fixed $\theta \in T$, and since $t$ is arbitrary in $(0,1)$, we conclude that $u(z) \equiv 0$ on $\Omega$.

7. Let $G_{\Omega-\bar{\Omega}_{t}}(z, \zeta)$ be the Green's function of $\Delta u=P u$ on $\Omega-\bar{\Omega}_{t}$ and $H_{\Omega-\bar{\Omega}_{t}}(z, \zeta)$ the harmonic Green's function. We simply denote by $H(z, \zeta)$ the harmonic Green's function of $\Omega$ and hence of $|z|<1$, i.e.

$$
H(z, \zeta)=\log \left|\frac{1-\bar{\zeta} z}{z-\zeta}\right|
$$

by (3) we have

$$
0<G_{\Omega-\bar{\Omega}_{t}}(z, \zeta) \leq H_{\Omega-\bar{\Omega}_{t}}(z, \zeta) \leq H(z, \zeta) .
$$

Since $G_{\Omega-\bar{\Omega}_{t}}(z, \zeta) \leq G_{\Omega-\bar{\Omega}_{s}}(z, \zeta)$ for $0<s \leq t<1$, the Harnack principle assures the existence of

$$
G(z, \zeta)=\lim _{t \rightarrow 0} G_{\Omega-\bar{\Omega}_{t}}(z, \zeta)
$$

which will be referred to as the Green's function of $\Delta u=P u$ on $\Omega$.

Under the assumption that the limit (15) exists for every $u \in \mathscr{B}$ we next prove the existence of

$$
K(\zeta) \equiv \lim _{z \rightarrow 0} G(z, \zeta) / e(z) \in \mathscr{P}
$$

for every fixed $\zeta \in \Omega$ (cf. Heins [4], Hayashi [3]).

Suppose $\zeta \in \Omega-\bar{\Omega}_{t}(t \in(0,1))$ and let $c(\zeta)=\max _{\beta_{t}} G(\cdot, \zeta)$ and $c^{\prime}(\zeta)$ $=\min _{\beta_{t}} e$. Since $G(\cdot, \zeta)$ and $\left(c(\zeta) / c^{\prime}(\zeta)\right) e$ are in $\mathscr{B}_{t}$ and the former is dominated by the latter on $\beta_{t},\left\{G(z, \cdot) / e(z) ; z \in \Omega_{t}\right\}$ is a uniformly bounded family of positive solutions of $\Delta u=P u$ on $\Omega-\bar{\Omega}_{t}$ for every $t \in(0,1)$. Hence by the Harnack principle $\{G(z, \cdot) / e(z) ; z \rightarrow 0\}$ is a normal family on each compact set in $\Omega$. Contrary to the assertion assume the limit 
(16) does not exist. Then there exist two sequences $\left\{z_{j, n}\right\}(j=1,2)$ in $\Omega$ coverging to zero such that

$$
K_{j}(\zeta)=\lim _{n \rightarrow \infty} G\left(z_{j, n}, \zeta\right) / e\left(z_{j, n}\right)
$$

exist on $\Omega(j=1,2)$ and $K_{1}(z) \not \equiv K_{2}(z)$ on $\Omega$. Clearly $K_{j} \in \mathscr{P} \quad(j=1,2)$. For any $u \in \mathscr{B}$ let $u_{t}=u_{1, t}$ as in (6) and $G_{t}(z, \zeta)=G_{\Omega-\bar{\Omega}_{t}}(z, \zeta)$. By (4)

$$
u_{t}(z)=-\frac{1}{2 \pi} \int_{0}^{2 \pi} u_{t}\left(e^{i \theta}\right)\left[\frac{\partial}{\partial r} G_{t}\left(z, r e^{i \theta}\right)\right]_{r=1} d \theta
$$

for $z \in \Omega-\bar{\Omega}_{t}$. On letting $t \rightarrow 0$ we have

$$
u(z)=-\frac{1}{2 \pi} \int_{0}^{2 \pi} u\left(e^{i \theta}\right)\left[\frac{\partial}{\partial r} G\left(z, r e^{i \theta}\right)\right]_{r=1} d \theta
$$

for every $z \in \Omega$ and a fortiori

$$
\frac{u\left(z_{j, n}\right)}{e\left(z_{j, n}\right)}=-\frac{1}{2 \pi} \int_{0}^{2 \pi} u\left(e^{i \theta}\right)\left[\frac{\partial}{\partial r}\left(\frac{G\left(z_{j, n}, r e^{i \theta}\right)}{e\left(z_{j, n}\right)}\right)\right]_{r=1} d \theta
$$

for $j=1,2$. By (15), on letting $n \rightarrow \infty$, we have

$$
\ell(u) / \ell(e)=-\frac{1}{2 \pi} \int_{0}^{2 \pi} u\left(e^{i \theta}\right)\left[\frac{\partial}{\partial r} K_{j}\left(r e^{i \theta}\right)\right]_{r=1} d \theta
$$

for $j=1,2$. On setting $L(z)=K_{1}(z)-K_{2}(z)$, we conclude that

$$
\int_{0}^{2 \pi} u\left(e^{i \theta}\right)\left[\frac{\partial}{\partial r} L\left(r e^{i \theta}\right)\right]_{r=1} d \theta=0
$$

for every $u \in \mathscr{B}$ and hence for every $u \in C(\beta)$. Thus

$$
L\left(e^{i \theta}\right)=\left[\frac{\partial}{\partial r} L\left(r e^{i \theta}\right)\right]_{r=1}=0
$$

on $T$ and therefore, by no. $6, L(z) \equiv 0$, i.e. $K_{1}(z) \equiv K_{2}(z)$, a contradiction.

8. Under the assumption (16) we finally conclude that any function $u(z)$ in $\mathscr{P}$ is a constant multiple of $K(z)$, i.e. $\operatorname{dim} P=1$ and thus the Picard principle is valid (cf. Martin [8], Nakai [11], S. Itô [5], etc.).

For any $u$ in $\mathscr{P}$, let $\hat{u}_{t, s}$ be the solution of $\Delta u=P u$ on $\Omega_{t}-\bar{\Omega}_{s}$ $(0<s<t<1)$ with boundary values $u$ on $\beta_{t}$ and zero on $\beta_{s}$ : Let $G_{s}(z, \zeta)=G_{\Omega-\bar{\Omega}_{s}}(z, \zeta)$. Fix a $z \in \Omega-\bar{\Omega}_{t}$. The Green formula applied to $u$ and $G(z, \cdot)$ for the region $\Omega-\bar{\Omega}_{t}$ yields 


$$
2 \pi u(z)=-\int_{\beta_{t}} G(z, \zeta) \frac{\partial}{\partial \nu_{\zeta}} u(\zeta) d s_{\zeta}+\int_{\beta_{t}} u(\zeta) \frac{\partial}{\partial \nu_{\zeta}} G(z, \zeta) d s_{\zeta}
$$

and also to $G_{s}(z, \cdot)$ and $\hat{u}_{t, s}$ for the region $\Omega_{t}-\bar{\Omega}_{s}$ with making $s \rightarrow 0$ yields

$$
0=-\int_{\beta_{t}} G(z, \zeta) \frac{\partial}{\partial \nu_{\zeta}} \hat{u}_{t}(\zeta) d s_{\zeta}+\int_{\beta_{t}} u(\zeta) \frac{\partial}{\partial \nu_{\zeta}} G(z, \zeta) d s_{\zeta}
$$

where $\hat{u}_{t}=\lim _{s \rightarrow 0} \hat{u}_{t, s} \in \mathscr{B}_{t}$ with $\hat{u}_{t}=u$ on $\beta_{t}$. Subtraction of the latter from the former in the above two identities gives

$$
u(z)=\frac{1}{2 \pi} \int_{0}^{2 \pi} G\left(z, t e^{i \theta}\right)\left[\frac{\partial}{\partial r}\left(\hat{u}_{t}\left(r e^{i \theta}\right)-u\left(r e^{i \theta}\right)\right)\right]_{r=t-0} t d \theta .
$$

Since $u\left(r e^{i \theta}\right)-\hat{u}_{t}\left(r e^{i \theta}\right) \geq 0$ on $\Omega_{t}$ and zero on $\beta_{t}$,

$$
d \mu_{t}(\theta)=\frac{1}{2 \pi} e\left(t e^{i \theta}\right)\left[\frac{\partial}{\partial r}\left(u_{t}\left(r e^{i \theta}\right)-u\left(r e^{i \theta}\right)\right)\right]_{r=t-0} t d \theta \geq 0
$$

on $U$. Let

$$
K(z, \zeta)=G(z, \zeta) / e(\zeta)
$$

By (16), $K(z, \zeta)$, as a function of $\zeta$, is continuous on $(|\zeta|<1)-\{z\}$ and $K(z, 0)=K(z)$, and we have

$$
u(z)=\int_{0}^{2 \pi} K\left(z, t e^{i \theta}\right) d \mu_{t}(\theta)
$$

for $z \in \Omega-\bar{\Omega}_{t}$. Fix a $t_{0} \in(0,1)$ and a $z_{0} \in \Omega-\bar{\Omega}_{t_{0}}$. Since $K\left(z_{0}, \zeta\right)$, as a function of $\zeta$, is a bounded solution of (12) on $\Omega_{t_{0}}$, (13) implies that

$$
a=\inf _{a_{0}} K\left(z_{0}, \zeta\right)>0
$$

Set $c_{t}=\int_{0}^{2 \pi} d \mu_{t}(\theta)$. Then $0 \leq c_{t} \leq u\left(z_{0}\right) / a$ for $t \in\left(0, t_{0}\right)$, and thus we can find a decreasing sequence $\left\{t_{n}\right\} \subset\left(0, t_{0}\right)$ coverging to zero such that $c=\lim _{n \rightarrow \infty} c_{t_{n}}$ exists. Hence by

$$
u(z)=\int_{0}^{2 \pi}\left(K\left(z, t e^{i \theta}\right)-K(z)\right) d \mu_{t_{n}}(\theta)+c_{t_{n}} K(z),
$$

we deduce

$$
|u(z)-c K(z)| \leq c_{t_{n}} \sup _{|\zeta|<t_{n}}|K(z, \zeta)-K(z, 0)|+\left|c_{t_{n}}-c\right| \cdot K(z)
$$

for $z \in \Omega-\bar{\Omega}_{t_{n}}$. On letting $n \rightarrow \infty$, we conclude that $u(z)=c K(z)$ on $\Omega$. 
9. We are ready to proceed to the proof of our theorem. All we have to prove is that the condition (1) implies (14), i.e. $\ell(e)>0$. Then, by no. 5 , (15) is valid and a fortiori (16) follows by no. 7, which in turn implies $\operatorname{dim} P=1$ by no. 8 .

Let $\left\{r_{n}\right\}$ be a decreasing sequence in $(0,1)$ converging to zero and $\varepsilon_{n}$ be such that $\left(r_{n+1}+r_{n}\right) / 2<r_{n}-\varepsilon_{n}<r_{n}+\varepsilon_{n}<\left(r_{n}+r_{n-1}\right) / 2$ for $n=1,2, \ldots$ with $r_{0}=1$ and that

$$
\int_{A_{n}} P(z) \log \frac{1}{|z|} d x d y<2^{-n}, \quad A_{n}=\left\{r_{n}-\varepsilon_{n}<|z|<r_{n}+\varepsilon_{n}\right\} .
$$

This can be achieved by taking $\varepsilon_{n}>0$ sufficiently small. Replacing $E$ in (1) by $E-\bigcup_{n=1}^{\infty} A_{n}$ we can thus assume

$$
E \cap \beta_{r_{n}}=\phi \quad(n=1,2, \cdots) .
$$

We denote by $e_{n}$ the $P$-unit on $\Omega_{r_{n}}$. Let $\left\{S_{n, m}\right\}$ be an increasing sequence $(m=1,2, \ldots)$ of subregions $S_{n, m}$ of $\Omega_{r_{n}}-E$ such that $\partial S_{n, m}$ consists of a finite number of disjoint Jordan curves with $\beta_{r_{n}}$ a component of $\partial S_{n, m}$ and $\cup_{m=1}^{\infty} S_{n, m}=\Omega_{r_{n}}-E$. We denote by $u_{n, m}\left(h_{m, n}\right.$, resp.) the solution of $\Delta u=P u$ (the harmonic function, resp.) on $S_{n, m}$ with boundary values 1 on $\beta_{r_{n}}$ and zero on $\partial S_{n, m}-\beta_{r_{n}}$. Let $H_{n, m}(z, \zeta)$ be the harmonic Green's function of $S_{n, m}$. Then $H_{n}(z, \zeta)=\lim _{m \rightarrow \infty} H_{n, m}(z, \zeta)$ is the harmonic Green's function of $\Omega_{r_{n}}-E$. By (2)

$$
h_{n, m}(z)=u_{n, m}(z)+\frac{1}{2 \pi} \int_{S_{n, m}} H_{n, m}(z, \zeta) P(\zeta) u_{n, m}(\zeta) d \xi d \eta
$$

Since $\left\{h_{n, m}\right\}\left(\left\{u_{n, m}\right\}\right.$, resp.) is increasing $(m=1,2, \cdots), h_{m}=\lim _{m \rightarrow \infty} h_{n, m}$ $\left(u_{n}=\lim _{m \rightarrow \infty} u_{n, m}\right.$, resp.) is a bounded harmonic function (a bounded solution of $\Delta u=P u$, resp.) on $\Omega_{r_{n}}-E$ with boundary values 1 on $\beta_{r_{n}}$. Moreover, since $H_{n, m}$ is increasing, the Lebesgue-Fatou theorem yields

$$
h_{n}(z)=u_{n}(z)+\frac{1}{2 \pi} \int_{\Omega_{r_{n}-E}} H_{n}(z, \zeta) P(\zeta) u_{n}(\zeta) d \xi d \eta
$$

Let $H(z, \zeta)=\log (|1-\bar{\zeta} z| /|z-\zeta|)$ be the harmonic Green's function on $\Omega$ and hence on $|z|<1$. Observe that $u_{n} \leq e_{n} \leq 1$ and $H_{n}(z, \zeta) \leq H(z, \zeta)$. Therefore

$$
h_{n}(z) \leq e_{n}(z)+\frac{1}{2 \pi} \int_{\Omega_{r_{n}-E}} H(z, \zeta) P(\zeta) d \xi d \eta
$$


for $z \in \Omega_{r_{n}}$ where we set $h_{n}=0$ on $E$. On integrating both sides of (17) on the circle $|z|=r \in\left(0, r_{n}\right)$ and using the Fubini theorem and the circle mean formula of Green's function:

$$
\frac{1}{2 \pi} \int_{0}^{2 \pi} H\left(r e^{i \theta}, \zeta\right) d \theta=\min \left(\log \frac{1}{r}, \log \frac{1}{|\zeta|}\right) \leq \log \frac{1}{|\zeta|},
$$

we deduce (cf. no. 3)

$$
h_{n}^{*}(r) \leq e_{n}^{*}(r)+\frac{1}{2 \pi} \int_{\Omega_{r_{n}}-E} P(\zeta) \log \frac{1}{|\zeta|} d \xi d \eta
$$

10. By comparing the boundary values we see that $h_{n+1, m} \geq h_{n, k}$ on $S_{n+1, m} \cap S_{n, k}$ if $m$ is sufficiently large for any fixed $k$. Therefore $h_{n+1}$ $\geq h_{n}$ on $\Omega_{r_{n+1}}$ and a fortiori $h_{n+1}^{*} \geq h_{n}^{*}$ on $\left(0, r_{n+1}\right]$ for $n=1,2, \ldots$. It is also clear that $e_{n+1}^{*} \geq e_{n}^{*}$ on $\left(0, r_{n+1}\right]$ for $n=1,2, \cdots$. Since we have set $h_{n}=0$ on $E, h_{n}$ is subharmonic on $\Omega_{r_{n}}$, and clearly $e_{n}$ is subharmonic on $\Omega_{r_{n}}$. Therefore

$$
a_{n}=\lim _{r \rightarrow 0} h_{n}^{*}(r) \geq 0, \quad b_{n}=\lim _{r \rightarrow 0} e_{n}^{*}(r) \geq 0
$$

exist (cf. no. 3) and $a_{n} \leq a_{n+1} \leq 1$ and $b_{n} \leq b_{n+1} \leq 1$ for $n=1,2, \cdots$, and thus

$$
a=\lim _{n \rightarrow \infty} a_{n} \in[0,1], \quad b=\lim _{n \rightarrow \infty} b_{n} \in[0,1]
$$

exist. By (18) we have

$$
a_{n} \leq b_{n}+\frac{1}{2 \pi} \int_{\Omega_{n_{n}-E}} P(\zeta) \log \frac{1}{|\zeta|} d \xi d \eta
$$

In view of (1) we have

$$
\lim _{n \rightarrow \infty} \frac{1}{2 \pi} \int_{\Omega_{r_{n}-E}} P(\zeta) \log \frac{1}{|\zeta|} d \xi d \eta=0
$$

and finally we conclude that $a \leq b$, i.e.

$$
\lim _{n \rightarrow \infty}\left(\lim _{r \rightarrow 0} h_{n}^{*}(r)\right) \leq \lim _{n \rightarrow \infty}\left(\lim _{r \rightarrow 0} e_{n}^{*}(r)\right) .
$$

11. Since $h_{n}>0$ on $\Omega_{r_{n}}-E$ and $z=0$ is an irregular boundary point of $\Omega-E$ and hence of $\Omega_{r_{n}}-E$, the Bouligand criteriond assures that 


$$
\lim _{z \in \Omega_{r_{n}}-E, z \rightarrow 0} h_{n}(z)>0 \text {. }
$$

On the other hand $h_{n}$ is subharmonic on $\Omega_{r_{n}}$ by the fact that we have defined $h_{n}=0$ on $E$, and therefore (cf. no. 3)

$$
a_{n}=\lim _{r \rightarrow 0} h_{n}^{*}(r)=\lim _{z \rightarrow 0} \sup h_{n}(z)=\limsup _{z \in \Omega_{r_{n}}-E, z \rightarrow 0} h_{n}(z)>0
$$

for every $n=1,2, \cdots$. Since $a_{n} \leq a_{n+1}$, we conclude that $a=\lim _{n \rightarrow \infty} a_{n}$ $>0$ and by (19) $\lim _{n \rightarrow \infty}\left(\lim _{r \rightarrow 0} e_{n}^{*}(r)\right)>0$. Thus there exists an $n$ such that

$$
\lim _{r \rightarrow 0} e_{n}^{*}(r)>0
$$

Let $c=\inf _{\beta_{n}} 1 / e>0$. Then $e_{n} \leq c e$ on $\beta_{r_{n}}$ implies that $e_{n} \leq c e$ on $\Omega_{r_{n}}$ and thus $e_{n}^{*} \leq c e^{*}$. Therefore

$$
c \ell(e)=\ell(c e)=\lim _{r \rightarrow 0}(c e)^{*}(r)=\lim _{r \rightarrow 0} c e^{*}(r) \geq \lim _{r \rightarrow 0} e_{n}^{*}(r)>0,
$$

i.e. we have shown that $\ell(e)>0$, i.e. (14) is valid.

The proof of the theorem is herewith complete.

12. At the end we state several important open problems related to elliptic dimensions. Let $P$ and $Q$ be densities on $0<|z| \leq 1$ and $c \geq 1$ a real number. We ask:

Problem 1. Is the relation $\operatorname{dim} c P=\operatorname{dim} P$ valid;

Problem 2. Does the inequality $P \leq Q \operatorname{imply} \operatorname{dim} P \leq \operatorname{dim} Q$ ?

In the affirmative case we can deduce the important order comparison theorem: If $c^{-1} P \leq Q \leq c P$ then $\operatorname{dim} P=\operatorname{dim} Q$, which is in question at present. These problems should also be asked for densities on Riemann surfaces (cf. Royden [17], Nakai [12], Lathtinen [7], etc.).

If we restrict ourselves to rotation free densities $P$ on $0<|z| \leq 1$, i.e. densities satisfying $P(z)=P(|z|)$ on $\Omega$, then we know that $\operatorname{dim} P$ is either 1 or the cardinal number $c$ of continuum and we have a complete criterion for $\operatorname{dim} P=1$ (cf. Nakai [13]). It is also instructive for the study of elliptic dimensions to observe the following example: For densities $P_{\lambda}(z)=|z|^{-\lambda}, \operatorname{dim} P_{\lambda}=1$ if $\lambda \in[-\infty, 2]$ and $\operatorname{dim} P_{\lambda}=\mathfrak{c}$ if $\lambda \in(2, \infty)$ where $P_{-\infty} \equiv 0$ (see [13]). Related to these we ask for general densities $P$ on $0<|z| \leq 1$ the following

Problem 3. How widely the range of $P \rightarrow \operatorname{dim} P$ can cover cardinals; 
Problem 4. What is the comprehensive complete condition for $\operatorname{dim} P$ $=1$ ?

These can also be discussed in the frame of Riemann surface setting, e.g. for densities on ends in the sense of Heins [4] (cf. Ozawa [15, 16], Myrberg [10], Kuramochi [6], Constantinescu-Cornea [2], Hayashi [3], etc.).

\section{REFERENCES}

[1] M. Brelot: Étude de l'équation de la chaleur $\Delta u=c(M) u(M), c(M)$, au voisinage d'un point singulier du coefficient, Ann. Ec. N. Sup., 48 (1931), 153-246.

[2] C. Constantinescu-A. Cornea: Über einige Problem von M. Heins, Rev. math. pures appl., 4 (1959), 277-281.

[3] K. Hayashi: Les solutions positives de l'équation $\Delta u=P u$ sur une surface de Riemann, Kōdai Math. Sem. Rep., 13 (1961), 20-24.

[ 4 ] M. Heins: Riemann surfaces of infinite genus, Ann. Math., 55 (1952), 296-317.

[5] S. Itô: Martin boundary for linear elliptic differential operators of second order in a manifold, J. Math. Soc. Japan, 16 (1964), 307-334.

[6] Z. Kuramochi: An example of a null-boundary Riemann surface, Osaka Math. J., 6 (1954), 83-91.

[ 7 ] A. Lahtinen: On the equation $\Delta u=P u$ and the classification of acceptable densities on Riemann surfaces, Ann. Acad. Sci. Fenn., 533 (1973), 1-26.

[8] R. Martin: Minimal positive harmonic functions, Trans. Amer. Math. Soc. 49 (1941).

[ 9 ] C. Miranda: Partial Differential Equations of Elliptic Type, Springer, 1970.

[10] L. Myrberg: Über die Existenz der Greenschen Funktion der Gleichung $\Delta u=c(P) u$ auf Riemannschen Flächen, Ann. Acad. Sci. Fenn., 170 (1954).

[11] M. Nakai: The space of nonnegative solutions of the equation $\Delta u=P u$ on a Riemann surface, Kōdai Math. Sem. Rep., 12 (1960), 151-178.

[12] M. Nakai: Order comparisons on canonical isomorphisms, Nagoya Math. J., 50 (1973), 67-87.

[13] M. Nakai: Martin boundary over an isolated singularity of rotation free density, J. Math. Soc. Japan, 26 (1974), 483-507.

[14] M. Ozawa: Classification of Riemann surfaces, Kōdai Math. Sem. Rep., 4 (1952), 63-76.

[15] M. Ozawa: Some classes of positive solutions of $\Delta u=P u$ on Riemann surfaces, I, Kõdai Math. Sem. Rep., 6 (1954), 121-126.

[16] M. Ozawa: Some classes of positive solutions of $\Delta u=P u$ on Riemann surfaces, II. Kōdai Math. Sem. Rep., 7 (1955), 15-20.

[17] H. Royden: The equation $\Delta u=P u$, and the classification of open Riemann surfaces, Ann. Acad. Sci. Fenn., 271 (1959).

[18] M. Tsuji: Potential Theory in Mordern Function Theory, Maruzen, 1959.

[19] K. Yosida: Functional Analysis, Springer, 1965.

\section{Nagoya University}

